\title{
Galaxies Detected by the Dwingeloo Obscured Galaxies Survey
}

\author{
A. J. Rivers ${ }^{1}$, P. A. Henning ${ }^{1}$ and R. C. Kraan-Korteweg ${ }^{2}$ \\ 1 Department of Physics and Astronomy, University of New Mexico, \\ 800 Yale NE, Albuquerque, NM 87131, USA \\ andyr@as.unm.edu, henning@as.unm.edu \\ 2 Dept. de Astronomia, Universidad de Guanajuato, Mexico \\ kraan@astro.ugto.mx \\ Received 1998 November 9, accepted 1999 February 1
}

\begin{abstract}
The Dwingeloo Obscured Galaxies Survey (DOGS) is a 21-cm blind survey for galaxies hidden in the northern 'Zone of Avoidance' (ZOA): the portion of the optical extragalactic sky which is obscured by dust in the Milky Way. Like the Parkes southern hemisphere ZOA survey, the DOGS project is designed to reveal hidden dynamically important nearby galaxies and to help 'fill in the blanks' in the local large scale structure. To date, 36 galaxies have been detected by the Dwingeloo survey; 23 of these were previously unknown [no corresponding sources recorded in the NASA Extragalactic Database (NED)]. Among the interesting detections are three nearby galaxies in the vicinity of NGC 6946 and 11 detections in the Supergalactic plane crossing region. VLA follow-up observations have been conducted for several of the DOGS detections.
\end{abstract}

Keywords: surveys-galaxies: general

\section{Introduction}

Approximately $25 \%$ of the optical extragalactic sky is obscured by the dust and high stellar density of the Milky Way (Figure 1). Although diligent optical and infrared searches for galaxies narrow this 'Zone of Avoidance', in the most heavily obscured regions near the Galactic plane, only radio surveys consistently reveal hidden galaxies. Radio galaxy searches near the Galactic plane can help to complete our knowledge of the nearby large scale structure and explore the connectivity of superclusters and voids across the $\mathrm{ZOA}$.

A full survey of the northern ZOA has been recently completed using the Dwingeloo 25-m radiotelescope and the Westerbork array in total power mode. The survey region $\left(30^{\circ} \leq \ell \leq 220^{\circ} ;|b| \leq 5.25\right.$; $\left.0 \leq V_{\mathrm{LSR}} \leq 4000 \mathrm{~km} \mathrm{~s}^{-1}\right)$ was initially covered in a shallow search by the 25 -m ( 5 min per pointing; rms noise per channel $\sigma_{\mathrm{ch}}=175 \mathrm{mJy}$ ) for nearby, massive galaxies (Henning et al. 1998). Recently, a deeper survey sensitive to nearby dwarfs and to normal spirals at the survey limit has been completed by the $25-\mathrm{m}$ and the Westerbork array in total power mode. Results from the portion of the deep survey completed by the $25-\mathrm{m}$ are reported here. Interesting regions covered by DOGS include a portion of the Local Void, the nearby IC342/Maffei group of galaxies and the region of sky where the Local Supercluster crosses the Galactic plane.

\section{Telescope Parameters and Search Strategy}

The 25-m Dwingeloo telescope operating at 21-cm has a half-power-beamwidth (HPBW) of $0^{\circ} \cdot 6$ which may be thought of as the survey resolution. A DAS-1000 channel autocorrelator spectrometer is utilised in the telescope backend; the rms noise per channel is typically $\sigma_{\mathrm{ch}}=40 \mathrm{mJy}$ for a $1 \mathrm{hr}$ integration. Each DOGS observation consists of a sequence of five contiguous pointings at constant Galactic latitude (Figure 2). Five On-Off pairs created from the sequence ensure that a real galaxy will appear twice, once as a positive signal, and again as a negative one, referenced against two independent scans. Overlapping the constant latitude grids to form a honeycombed coverage of the sky allows for detection of galaxies in adjacent pointings and facilitates a more accurate determination of their positions. A similar observing strategy was employed during the Westerbork portion of the survey; beam size and velocity coverage were identical and a similar survey sensitivity achieved. A 15 pointing grid was used for efficiency reasons, but the core On-Off pair strategy remained the same. The complete survey incorporates approximately 15,000 partially overlapping pointings.

\section{Results}

Approximately $60 \%$ of the DOGS survey was completed using the Dwingeloo 25-m telescope. 


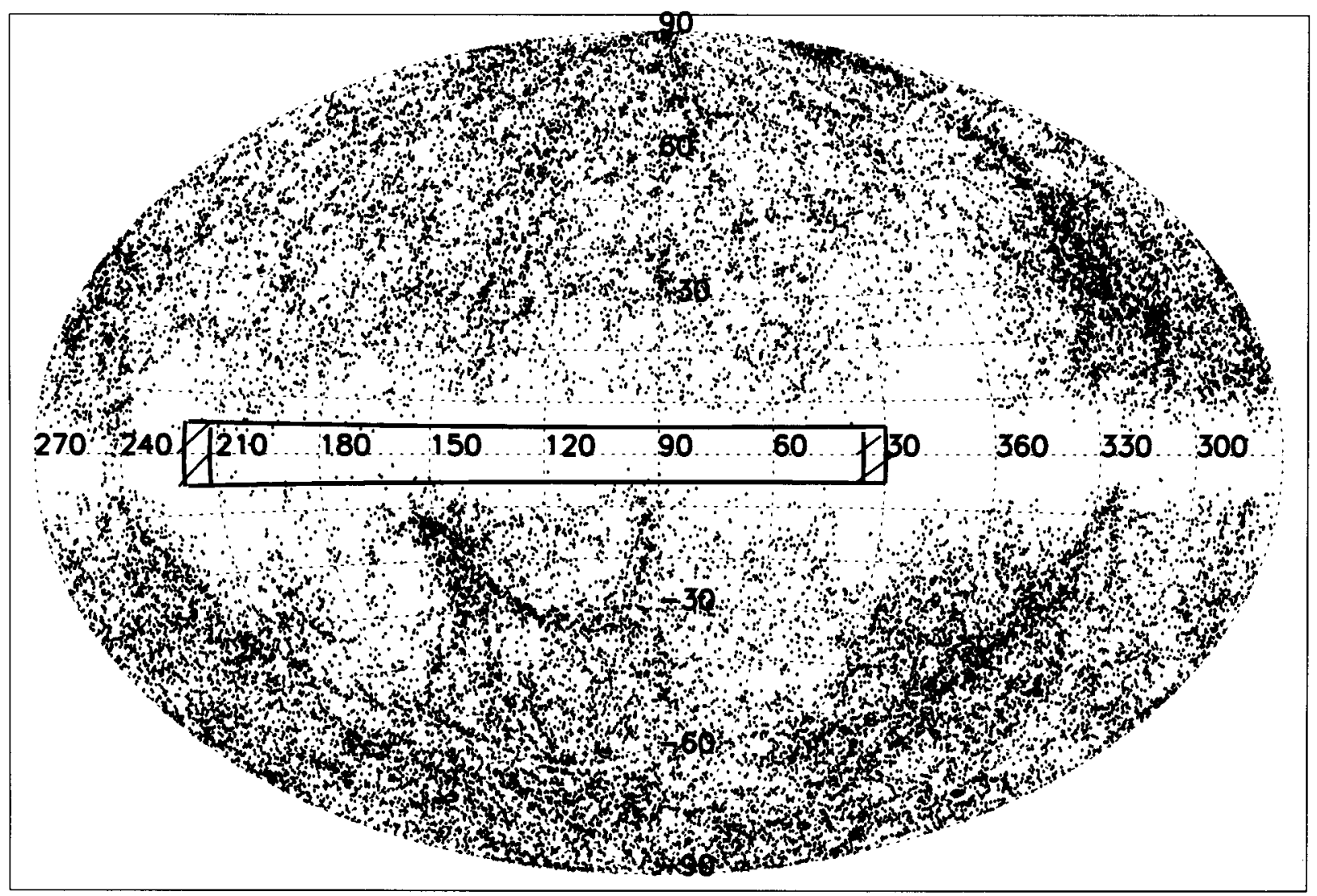

Figure 1-A combined Galactic longitude versus latitude plot of approximately 30000 galaxies collected from the MCG, UGC and ESO optical catalogues shows the 'Zone of Avoidance' caused by dust in the Galactic plane. The spatial region covered by the Dwingeloo survey is indicated. The hatched region represents the spatial overlap with the Parkes ZOA survey.

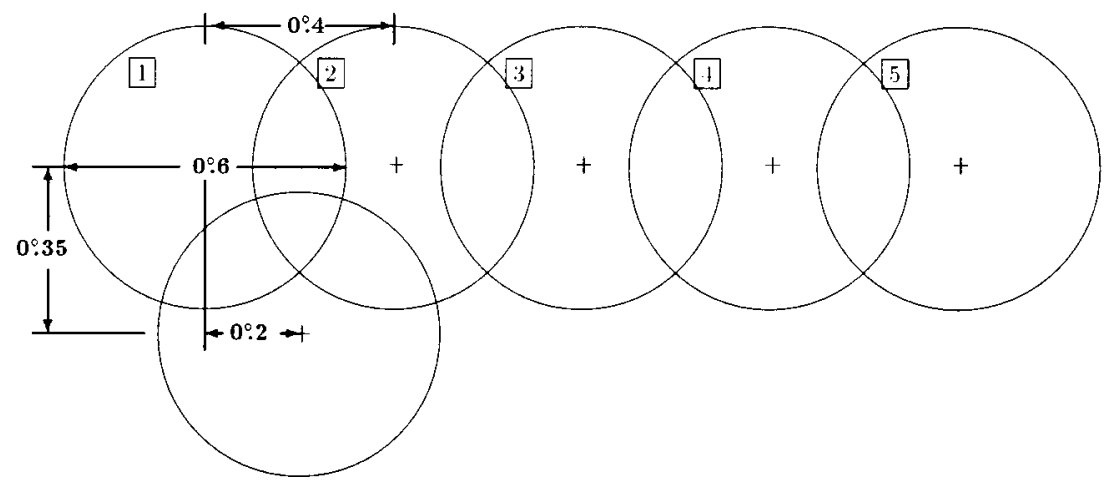

Figure 2-Distribution of survey grid points. Galactic longitude spacing is $\Delta \ell=0.4$ while the successive rows of constant Galactic latitude are separated by $\Delta b=0^{\circ} \cdot 35$. Each grid point is marked with a + surrounded by a circle indicating the HPBW of 0.6 .

In this portion of the survey, 36 galaxies were confirmed, 23 of which were previously unknown (no NED counterpart). The number of galaxies detected is consistent with calculations based on an assumed Hi mass function (Zwaan, Briggs \& Sprayberry 1997) and the survey sensitivity which predict between 50 and 100 detections within the survey range. Recent Westerbork observations completed the survey and galaxies discovered will soon be incorporated into the Dwingeloo sample (cf. Figure 3 for the location of detected galaxies).
Five of the 36 sources were originally identified by the shallow survey including Dwingeloo 1 and Maffei 2, both members of the nearby Maffei/IC 342 group of galaxies (Kraan-Korteweg et al. 1994). During the deep survey another known group member, MB 1 (McCall \& Buta 1995), was identified and two additional members still await confirmation observations.

The most significant nearby, previously unknown galaxy identified by DOGS was Dwingeloo 1. Given the $80 \%$ coverage of the survey region by the shallow 


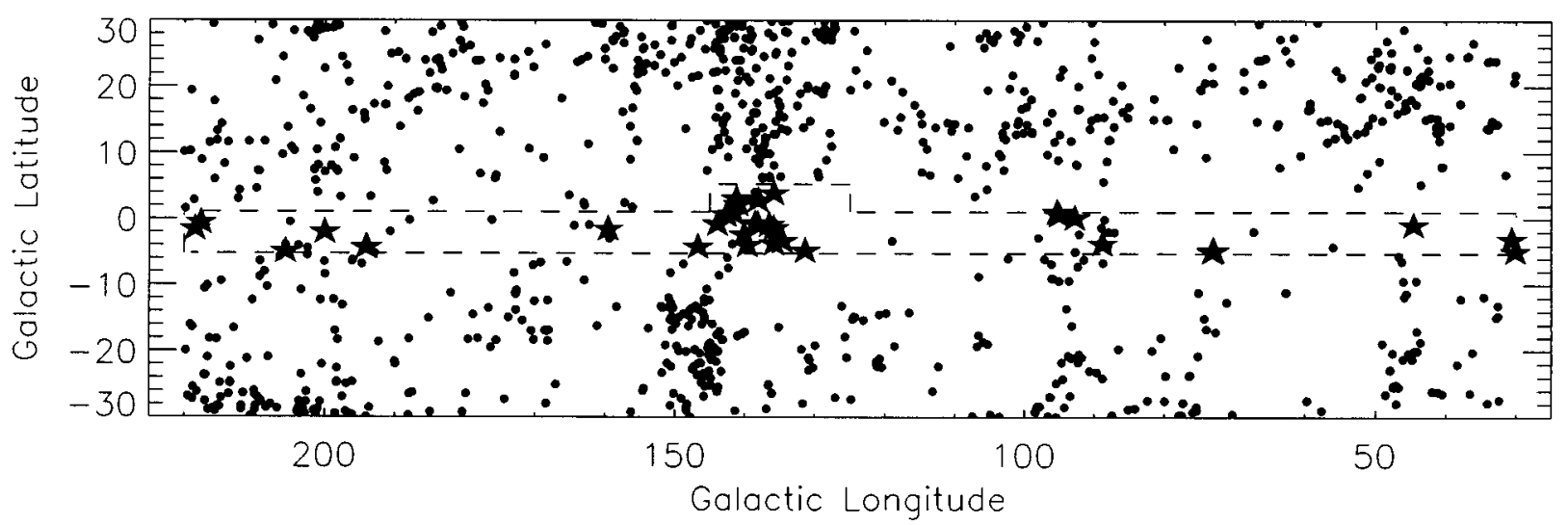

Figure 3-Spatial distribution of DOGS sources (indicated by $\star$ ) combined with Lyon-Meudon Extragalactic Database (LEDA) galaxies out to $V_{\mathrm{LSR}} \leq 4000 \mathrm{~km} \mathrm{~s}^{-1}(\bullet)$. The $60 \%$ of the survey covered by the $25-\mathrm{m}$ telescope and analysed in this paper is shown by the dashed line.
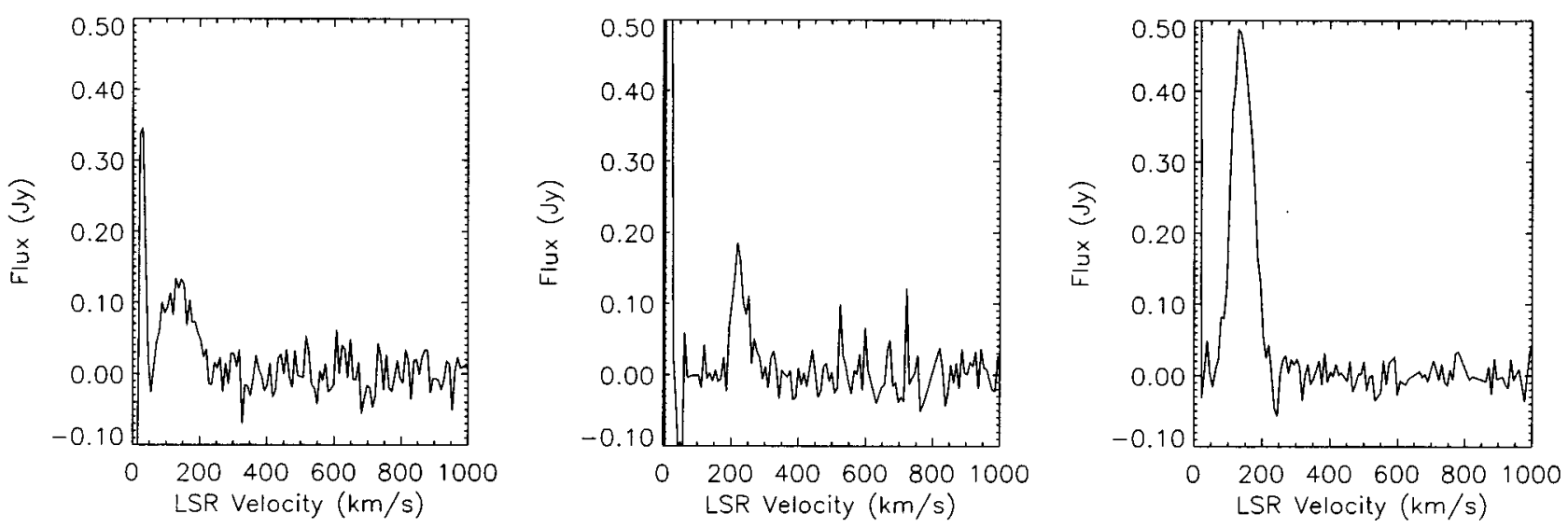

Figure 4-Spectra from the Dwingeloo 25-m telescope of three galaxies found in the vicinity of NGC 6946. Pictured are, from left to right, IRAS 21189+4503, Dw092 $8+0 \cdot 5$ and Dw095 $0+1 \cdot 0$.

Table 1. Possible members of the NGC 6946 group

Hi masses are calculated based on the assumption that all galaxies lie at the same distance as NGC 6946 (6 Mpc, Sharina, Karachentsev \& Tikhonov 1997). The Hi mass of NGC 6946 is based on the mean of the six flux entries in LEDA which are corrected for the beam-filling effect. The uncertainty in the masses of the three Dwingeloo detected sources is primarily due to the uncertainty in the positions of the galaxies

\begin{tabular}{lcrccl}
\hline \multicolumn{1}{c}{ Galaxy name } & $\begin{array}{c}\ell \\
(\mathrm{deg} .)\end{array}$ & $\begin{array}{c}b \\
(\mathrm{deg} .)\end{array}$ & $\begin{array}{c}V_{\mathrm{LSR}} \\
\left(\mathrm{km} \mathrm{s}^{-1}\right)\end{array}$ & $\begin{array}{c}\log \left(M_{\mathrm{HI}}\right) \\
\left(M_{\odot}\right)\end{array}$ & \multicolumn{1}{c}{ Reference } \\
\hline HKK L150 & $96 \cdot 03$ & $12 \cdot 35$ & 132 & $7 \cdot 1$ & Huchtmeier et al. (1997) \\
UGC 11583 & $95 \cdot 63$ & $12 \cdot 31$ & 127 & $8 \cdot 2$ & Huchtmeier et al. (1997) \\
HKK L149 & $95 \cdot 57$ & $12 \cdot 22$ & 126 & $8 \cdot 1$ & Huchtmeier et al. (1997) \\
NGC 6946 & $95 \cdot 72$ & $11 \cdot 67$ & 48 & $10 \cdot 1$ & LEDA \\
Cepheus 1 & $94 \cdot 38$ & $8 \cdot 01$ & 65 & $9 \cdot 1$ & Burton et al. (1999) \\
Dw095.0+1.0 & $95 \cdot 05$ & $1 \cdot 16$ & 142 & $8 \cdot 6:$ & Henning et al. (1998) \\
Dw092 $\cdot 8+0 \cdot 5$ & $92 \cdot 82$ & $0 \cdot 53$ & 230 & $7 \cdot 8:$ & \\
IRAS 21189+4503 & $88 \cdot 85$ & $-3 \cdot 20$ & 228 & $8 \cdot 3:$ & Nakanishi et al. (1997) \\
\hline
\end{tabular}

survey (Henning et al. 1998), chances are low that a massive nearby spiral was missed, since nearby galaxies appear in many adjacent pointings, all of which would have to be missed for the galaxy to escape detection. Thus, it is fairly unlikely that there is another previously unidentified massive galaxy whose gravitational influence significantly impacts Local Group peculiar motion or internal dynamics in the area covered by the survey.

\subsection{Supergalactic Plane Crossing Region and Local Void}

Although the Dwingeloo survey is limited in survey depth $\left(V_{\mathrm{LSR}} \leq 4000 \mathrm{~km} \mathrm{~s}^{-1}\right)$, two primary large scale structures fall partially within the survey range: the Local Void and the Local Supercluster. Eleven galaxies were discovered in the survey coverage of the Local Supercluster crossing region $\left(\ell \sim 140^{\circ} ;|b| \leq\right.$ $5.25)$; six of these sources are noted in NED. Known 


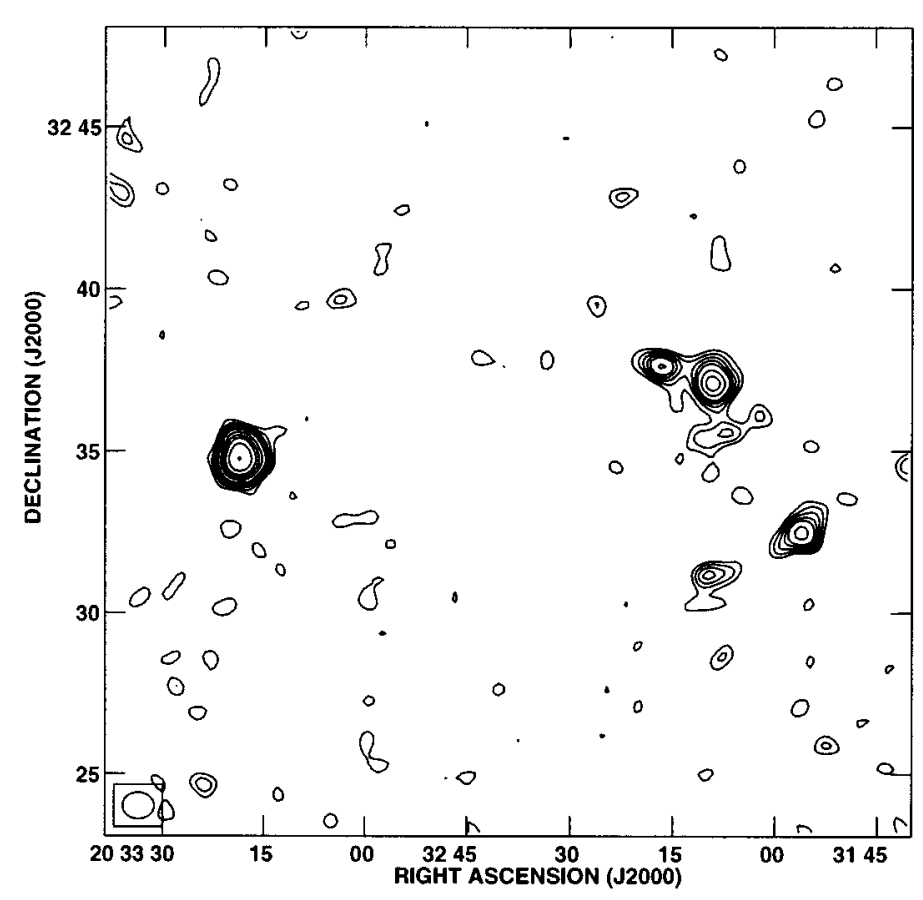

Figure 5-A synthesis map of DOGS detection Dw073 $2-4 \cdot 2$ from the VLA. A galaxy group is revealed in a sample velocity slice $\left(V_{\mathrm{LSR}}=3219\right.$ $\mathrm{km} \mathrm{s}^{-1}, \Delta v \simeq 10 \mathrm{~km} \mathrm{~s}^{-1}$ ).

structures appear continuous and well defined across the Galactic plane with a narrow bridge of galaxies visible at $\ell \sim 142^{\circ}$ and $V_{\mathrm{LSR}} \sim 1400 \mathrm{~km} \mathrm{~s}^{-1}$.

Near the Local Void $\left(\ell \sim 33^{\circ}, b \sim-15^{\circ}\right)$, Marzke, Huchra \& Geller (1996) and Roman et al. (1998) found evidence for a nearby cluster at $\sim 1500 \mathrm{~km} \mathrm{~s}^{-1}$. Two previously unknown galaxies were detected in this region, adding support for the hypothesised overdensity. These galaxies, Dw030.6-2.4 ( $\ell=$ $\left.30 \cdot 60, b=-2 \cdot 48, \quad V_{\mathrm{LSR}}=1480 \mathrm{~km} \mathrm{~s}^{-1}\right)$ and $\operatorname{Dw} 030 \cdot 1-4 \cdot 3\left(\ell=30 \cdot 09, \quad b=-4 \cdot 35, V_{\mathrm{LSR}}=\right.$ $1528 \mathrm{~km} \mathrm{~s}^{-1}$ ), were independently identified by the Parkes ZOA survey (Henning et al. 1999, present issue p. 35).

\subsection{NGC 6946 Group}

Three dwarf galaxies were detected in the region of NGC $6946(\ell=95 \cdot 72, b=11 \cdot 67)$ suggesting the possibility of a new nearby group. Of these, Dw095 $0+1 \cdot 0$ was originally recorded as a compact high velocity cloud (HVC) (Wakker 1990), but present data suggest it is in fact a nearby dwarf galaxy. With a velocity of $V_{\mathrm{GSR}}=368 \mathrm{~km} \mathrm{~s}^{-1}$, it has the highest redshift of any $\mathrm{HVC}$ in the Wakker catalog and the $50 \%$ velocity width of $\Delta V_{50}=100 \mathrm{~km}$ $\mathrm{s}^{-1}$ seen in the Dwingeloo spectrum is significantly broader than the $20-30 \mathrm{~km} \mathrm{~s}^{-1}$ velocity dispersion generally observed in HVCs. Naively assuming this galaxy is at the same distance as NGC 6946 ( 6 Mpc, Sharina, Karachentsev \& Tikhonov 1997) yields $M_{\mathrm{HI}} \simeq 4 \times 10^{8} M_{\odot}$. Also detected in the area were IRAS 21189+4503 (Nakanishi et al. 1997) and
Dw092 $8+0 \cdot 5$. Assuming a $6 \mathrm{Mpc}$ distance yields $M_{\mathrm{HI}} \simeq 2 \times 10^{8} M_{\odot}$ and $1 \times 10^{8} M_{\odot}$ respectively. The estimated neutral hydrogen masses are typical of dwarf galaxies, consistent with the gaussian shape of the 21-cm profiles (Figure 4). With the recent discovery of the LSB galaxy Cepheus 1 (Burton et al. 1999) seven galaxies with recessional velocites $V_{\mathrm{LSR}} \leq 250 \mathrm{~km} \mathrm{~s}^{-1}$ have been identified within $15^{\circ}$ of NGC 6946 (Table 1). If these galaxies do in fact signify a new nearby group, this group would lie some $40^{\circ}$ from the Supergalactic plane, considerably more than any other known group in the local universe. The potential exists for the discovery of additional galaxies in this group since the data recently collected by the Westerbork array for $+1^{\circ} \cdot 05 \leq b \leq+5.25$ have not yet been analysed.

\subsection{Results from Synthesis Follow-ups of DOGS Sources}

Synthesis observations of Dwingeloo galaxies were conducted with the VLA and WSRT in 1997. Snapshot WSRT observations yielded positions of DOGS sources though sensitivities were not adequate for detailed mapping. VLA follow-ups yielded a few interesting results. A galaxy group unresolved by the Dwingeloo 0.6 beam was resolved into five galaxies by follow-up VLA observations (Figure 5). Since early synthesis follow-ups to Parkes ZOA detections also have revealed interacting galaxy groups (StaveleySmith et al. 1998), it will be interesting to see if this is commonly seen in Hi selected samples. The total Hi mass measured for this group was $2 \times 10^{9} M_{\odot} h_{0}^{-2}$ 
with a mean velocity of $V_{\mathrm{LSR}}=3222 \mathrm{~km} \mathrm{~s}^{-1}$ and a calculated group diameter of approximately 150 $h_{0}^{-1} \mathrm{kpc}$. No optical counterparts were identified on POSS E plates under significant extinction $\left(A_{\mathrm{B}} \simeq\right.$ 4.5 mag from DIRBE reddening maps, Schlegel, Finkbeiner \& Davis 1998). A previously unknown low mass companion to IRAS $05596+1451$ was also discovered in a separate VLA synthesis map.

\section{Conclusions and Future Directions}

A wide field flux limited survey for $\mathrm{HI}$ luminous galaxies hidden in the northern ZOA has been completed. With approximately $60 \%$ of the data analysed we have detected 36 galaxies, 23 of which were previously unknown. Given the number found to date, in the full survey we expect to detect around 60 sources, which falls within our survey predictions of 50-100 galaxies.

Since the DOGS slice covers a wide range of the extragalactic sky including portions of the Local Supercluster and the Local Void, the collected sample will be well suited for a comparison study of galaxies in different environments.

Efforts are also underway to quantify the Galactic extinction, a poorly known quantity near the Galactic plane. Although the recently released DIRBE reddening maps (Schlegel, Finkbeiner \& Davis 1998) prove useful, they remain uncalibrated at low latitude $\left(|b| \leq 10^{\circ}\right)$. We are exploring the possibility of using near-infrared colours of galaxies from 2MASS data to independently determine the extinction and to thereby determine calibration constants and uncertainties for the DIRBE maps at low latitude.

\section{Acknowledgments}

We are grateful to A. Foley and D. Moorrees for their supervision of the 25-m day-to-day telescope operations, to T. Hess for the development of data translation routines, and to O. Lahav and W. B.
Burton for helpful discussions. We would also like to thank J. M. van der Hulst and R. Braun for their help in instigating and supporting the Westerbork portion of the DOGS project. The Dwingeloo 25-m radio telescope is supported by the Netherlands Foundation for Scientific Research (NWO). This research has made use of the NASA/IPAC Extragalactic Database (NED) and the Lyon-Meudon Extragalactic Database (LEDA). The research of P. A. Henning is supported by NSF Faculty Early Career Development (CAREER) Program award AST 95-02268.

\section{References}

Burton, W. B., Braun, R., Walterbos, R. A. M., \& Hoopes, C. G. 1999, AJ, in press

Henning, P. A., Kraan-Korteweg, R. C., Rivers, A. J., Loan, A. J., Lahav, O., \& Burton, W. B. 1998, AJ, 115, 584

Henning, P. A., Staveley-Smith, L., Kraan-Korteweg, R. C., \& Sadler, E. M. 1999, PASA, 16, 35

Huchtmeier, W. K., Karachentsev, I. D., \& Karachentseva, V. E. 1997, A\&A, 322, 375

Kraan-Korteweg, R. C., Loan, A. J., Burton, W. B., Lahav, O., Ferguson, H. C., Henning, P. A., \& Lynden-Bell, D. 1994, Nature, 372, 77

McCall, M. L., \& Buta, R. J. 1995, AJ, 109, 2460

Marzke, R. O., Huchra, J. P., \& Geller, M. J. 1996, AJ, 112,1803

Nakanishi, K., Takata, T., Yamada, T., Takeuchi, T., Shiroya, R., Miyazawa, M., Watanabe, S., \& Saito, M. 1997, ApJS, 112,245

Roman, A. T., Takeuchi, T., Nakanishi, K., \& Saito, M. 1998, PASJ, 50, 47

Schlegel, D. J., Finkbeiner, D. P., \& Davis, M. 1998, ApJ, 500,525

Sharina, M. E., Karachentsev, I. D., \& Tikhonov, N. A. 1997, Astron. Lett., 23, 373

Staveley-Smith, L., Juraszek, S., Koribalski, B. S., Ekers, R. D., Green, A. J., Haynes, R. F., Henning, P. A., Kesteven, M. J., Kraan-Korteweg, R. C., Price, R. M., Sadler, E. M. 1998, AJ, 116, 2717

Wakker, B. P. 1990, PhD thesis, Groningen Univ.

Zwaan, M., Briggs, F., \& Sprayberry, D. 1997, PASA, 14, 126 